\title{
Hospitals' Discharge Tendency and Risk of Death - An Analysis of 60,000 Norwegian Hip Fracture Patients
}

This article was published in the following Dove Press journal: Clinical Epidemiology

\author{
Sara Marie Nilsen ${ }^{1} *$ \\ Johan Håkon Bjørngaard (iD) $2,3, *$ \\ Fredrik Carlsen ${ }^{4}$ \\ Kjartan Sarheim Anthun ${ }^{2,5}$ \\ Lars Gunnar Johnsen ${ }^{6,7}$ \\ Lars Johan Vatten ${ }^{2}$ \\ Andreas Asheim (iD) 1,8 \\ 'Center for Health Care Improvement, \\ Trondheim University Hospital, \\ Trondheim, Norway; ${ }^{2}$ Department of \\ Public Health and Nursing, Norwegian \\ University of Science and Technology, \\ Trondheim, Norway; ${ }^{3}$ Faculty of Nursing \\ and Health Sciences, Nord University, \\ Levanger, Norway; ${ }^{4}$ Department of \\ Economics, Norwegian University of \\ Science and Technology, Trondheim, \\ Norway; ${ }^{5}$ Department of Health \\ Research, SINTEF Digital, Trondheim, \\ Norway; ${ }^{6}$ Department of Orthopaedic \\ Surgery, Trondheim University Hospital, \\ Trondheim, Norway; ${ }^{7}$ Department of \\ Neuromedicine, Norwegian University of \\ Science and Technology, Trondheim, \\ Norway; ${ }^{8}$ Department of Mathematical \\ Sciences, Norwegian University of \\ Science and Technology, Trondheim, \\ Norway
}

*These authors contributed equally to this work
Correspondence: Johan Håkon Bjørngaard NTNU, Institutt for samfunnsmedisin og sykepleie, Postboks 8905 , Trondheim 749I, Norway

Tel +47 92242734

$\mathrm{Fax}+4773597577$

Email johan.h.bjorngaard@ntnu.no
Purpose: A reduction in the length of hospital stay may threaten patient safety. This study aimed to estimate the effect of organizational pressure to discharge on 60-day mortality among hip fracture patients.

Patients and Methods: In this cohort study, hip fracture patients were analyzed as if they were enrolled in a sequence of trials for discharge. A hospital's discharge tendency was defined as the proportion of patients with other acute conditions who were discharged on a given day. Because the hospital's tendency to discharge would affect hip fracture patients in an essentially random manner, this exposure could be regarded as analogous to being randomized to treatment in a clinical trial. The study population consisted of 59,971 Norwegian patients with hip fractures, hospitalized between 2008 and 2016, aged 70 years and older. To calculate the hospital discharge tendency for a given day, we used data from all $5,013,773$ other acute hospitalizations in the study period.

Results: The probability of discharge among hip fracture patients increased by 5.5 percentage points $(95 \%$ confidence interval $(\mathrm{CI})=5.3-5.7)$ per 10 percentage points increase in hospital discharges of patients with other acute conditions. The increased risk of death that could be attributed to a discharge from organizational causes was estimated to 3.7 percentage points $(95 \% \mathrm{CI}=1.4-6.0)$. The results remained stable under different time adjustments, follow-up periods, and age cut-offs.

Conclusion: This study showed that discharges from organizational causes may increase the risk of death among hip fracture patients.

Keywords: length of stay, causality, bed occupancy, quality of healthcare, mortality, orthopedic procedures

\section{Plain Language Summary}

In Norway there are about 10,000 hip fractures per year. These patients have high mortality and often require lengthy hospitalizations. Hospital stays have been shortened for this and other patient groups. Whether this development has had negative effects is hard to investigate using standard statistical methods. For example, frail patients are likely to have longer hospital stays. Observed differences between patients with long and short stays might, therefore, reflect differences in the kind of patients that have long or short length of hospital stay. In this paper we suggest that rather than asking whether longer hospital stays are better or worse than shorter stays, one should ask what the effect of being discharged from organizational causes is. For example, this could be the difference in outcome for two patients who both were ready for discharge, but one was discharged to make room in the ward, while the other could stay. In this paper we attempted to capture such hypothetical situations by considering the increased chance of a patient being discharged when the rate of overall hospital discharges was high. Hospital discharges would for example be higher on 
Fridays, since staffing was often reduced at weekends. Each day was analysed as a new trial for discharge, thereby avoiding bias due to in-hospital deaths. The results show that there was a clear tendency to discharge more hip fracture patients with more hospital discharges. Our estimates show that discharges from organizational causes was associated with a 3.7 percentagepoint increased risk of death.

\section{Introduction}

Within many healthcare systems there are strong incentives to reduce length of hospitalization. Together with gradual but significant improvements in treatment, this has contributed to reduced length of stay for many conditions. ${ }^{1}$ However, concerns have been raised that shorter hospital stays may be a threat to patient safety. ${ }^{2,3}$ Frail hip fracture patients will often require lengthy hospitalizations due to multi-morbidity, and shorter stays among these patients may cause adverse outcomes.

It is known that hip fracture patients are operated on within settings that are under considerable pressure, often caused by the acute nature of the situation, and the limited availability of operating theaters and qualified staff. ${ }^{4}$ In a recent Swedish study ${ }^{5}$ hip fracture patients with hospitalizations of less than 10 days were found to have higher mortality compared to patients with longer stays. In contrast, a similar study from the US reported lower mortality associated with hospital stays of less than 10 days, compared to patients with longer stays. ${ }^{6}$

Such differences may, at least partly, be attributed to structural differences between healthcare systems, but methodological limitations of the studies may also result in discrepancies between findings. ${ }^{7}$ By comparing hip fracture patients according to length of hospitalization, confounding due to unmeasured patient characteristics may explain some of the differences between the studies. Another challenge is that many patients die while they are in hospital, which may introduce a biased association between length of stay and mortality. ${ }^{8}$ In previous studies ${ }^{5,6}$ mortality has been measured from time of discharge and, to be included in the analyses, patients had to survive until discharge, which is likely to introduce so-called immortal time bias. ${ }^{9}$ To reduce these potentials for bias, we treated the decision to discharge separately for each day in hospital instead of analysing length of stay.

Ideally, the decision to discharge is based on the patient's condition alone. However, for patients who are close to or ready for discharge, the decision can also be affected by external factors. ${ }^{10}$ If the clinical department is under pressure, for example in need of more available beds, it is possible that patients are discharged prematurely compared to discharges that take place on days with less pressure. In this study, we assumed that any external pressure to discharge would randomly affect each patient, and that would allow us to test whether expedited discharge could increase the risk of death. Contingent on a set of assumptions linked to the use of instrumental variable analyses, ${ }^{11,12}$ we estimated the causal effect of expedited discharge on the risk of death within 60 days among hip fracture patients.

\section{Methods}

\section{Study Cohort}

We used data from the Norwegian Patient Registry to acquire information about a nationwide cohort of 59,971 hip fracture patients (with 64,914 fractures) from 1 January 2008 to 31 December 2016. All Norwegian hospital trusts are required to submit information about their clinical activity to the national patient registry. The registry provides information on age and sex of the patient, as well as information on hospital, diagnosis, procedural codes, and time of admission and discharge for each hospital episode. Date of death was collected from the Norwegian Cause of Death Registry.

Patients admitted to hospital with a hip fracture were identified through a combination of ICD-10 codes and the Nordic Medico-Statistical Committee (NOMESCO) Classification of Surgical Procedures codes. ${ }^{13} \mathrm{We}$ included acute patients with ICD-10 codes S72.0x, S72.1x, or S72.2x (fracture of proximal femur) as primary diagnosis and one or more NOMESCO codes, NFBxy $(x=0-9, y=0-2$, primary prosthetic replacement of hip joint), or NFJxy $(x=0-9, y=0$ 2 , fracture surgery of femur) during their hospitalization. This definition has previously shown high accuracy in identifying hospitalizations for hip fractures while excluding stays due to rehabilitation. ${ }^{14}$ We also included hip fracture patients without the procedure codes who died in hospital, because they could have died before a required operational procedure. For patients who were hospitalized more than once within 30 days, we only included the first stay, and thus we avoided more than one entry for the same fracture. We included patients who were 70 years of age or older at admission. To calculate hospital discharge tendency for any given day we used data from all 5,013,773 acute hospitalizations, excluding hip fracture patients. See Supplementary Figure S1 for a flow chart of inclusion criteria.

The study was approved by the Regional Committee of Ethics in Medical Research (2016/2158-1). Patient consent was not required, as the regional ethical committee found that the conditions for exemption from the duty of 
confidentiality was met. The project was considered to be of significant importance for society, and the welfare and integrity of the patients were ensured. The data of this study are available from the Norwegian patient registry, but restrictions apply to the availability. Each patient has a unique, anonymous identification number throughout the observation period. These data were used under license for the current study and are not publicly available.

\section{Analytical Strategy}

In order to avoid selection due to in-hospital death we analyzed the observational cohort of hip fracture patients by mimicking a series of clinical trials. ${ }^{11,15}$ We analyzed outcomes for patients who were eligible for discharge on each day of hospitalization, where eligibility was defined as being alive and hospitalized. This procedure was followed for each day from day 2 until day 14 after admission, as only few patients remained hospitalized beyond day 14 . Withinperson correlation was accounted for since patients were included in one analysis for each day until discharge or death.

In an initial analysis we simply compared 60-day mortality of patients who were discharged on a given day with the corresponding outcomes of patients who were not discharged on that day. Because confounding by indication could be an issue using that approach, we performed an instrumental variable analysis to assess whether discharges from organizational causes could affect patient mortality. In the analysis, our candidate instrumental variable, the hospital's tendency to discharge, was defined as the proportion of all acute inpatients at the hospital who were discharged per given day, excluding hip fracture patients. The definition reflects the assumption that a hospital was under a certain pressure to discharge on any given day, and that this pressure was independent of the condition of the individual hip fracture patient. Therefore, such an extraneous pressure to discharge would most likely affect each patient in a random manner. To exemplify, on Fridays or days before a holiday, or on days when the hospital is in extra need for patient beds, the discharge tendency will be relatively higher than on other days.

Three basic assumptions ${ }^{16}$ must be satisfied for our estimate of the effect of expedited discharge to be valid: The instrumental variable, the hospital's tendency to discharge, has to be associated with the exposure (relevance), only affect the outcome through the likelihood of the exposure (exclusion restriction), and be independent of possible confounding factors (independence). The relevance assumption can be tested, and as a rule of thumb the association should at least have an F-statistic larger than 10 in order to avoid weak instrument bias. ${ }^{17}$ We cannot prove that the exclusion restriction and independence assumptions hold. However, sensitivity tests can provide evidence against or for their plausibility. These different sensitivity analyses are presented in detail.

\section{Statistical Analysis}

In the initial analysis, we used a Cox proportional hazards model to study the association between discharge (yes/no) on a given day and patient mortality.

Subsequently, we analyzed the association between hospital discharge tendency, i.e., an extraneous pressure to discharge, and patient mortality. This analytical approach is analogous to an intention to treat analysis in a clinical trial. We used time from admission as the time scale, and patients were followed for 60 days after being eligible for discharge or until death, whichever occurred first.

We computed the F-statistic of the association between hospital discharge tendency and discharge of hip fracture patients using ordinary least squares regression with bootstrapped confidence intervals to test the instrument relevance criterion. ${ }^{16}$ Instrument relevance means, in this case, that hip fracture patients have a higher propensity for being discharged on days with high discharge tendency.

In the full instrumental variable analysis, we used the hospital discharge tendency to predict discharge of hip fracture patients, and used this prediction to estimate the effect on risk of death within 60 days. The estimation was done using two-stage ordinary least squares ${ }^{18}$ (ivregress in Stata). To gain statistical power we aggregated all 13 trials (one for each day from day 2 to 14) into one overall estimate. We also estimated effects for triplets of days (day $2-4$, day $3-5$, etc.). The estimates were adjusted for hospital discharge tendency on all previous days of hospitalization to take into account possible differences in the population of eligible patients due to discharges on previous days. ${ }^{19}$ We used a robust variance estimator to account for within-person correlation.

In all analyses, patients were compared within the same month, year, and hospital to avoid confounding by differences between institutions, and possible seasonal and organizational factors. We adjusted for age and age as a quadratic term, sex, fracture of the femoral neck (S72.0), weekend or holiday admission, a dummy variable for day of hospitalization, and previous hospitalizations within the last two calendar months. The choice to follow patients for 60 days was made to capture a period when mortality was more likely to be affected by treatment episode than by other causes. ${ }^{20}$ 


\section{Instrumental Variable Assumption and Additional Analyses}

One central assumption in the analysis, the instrument independence assumption, was that hospital discharge tendency of other acute patients should not have any common causes of with death among the hip fracture patients. To investigate this assumption, we analysed possible associations between discharge tendency and known and measured risk factors for the prognosis of hip fracture patients (age, sex, type of fracture S72.0, and prior admissions).

To investigate whether the discharge tendency could affect mortality through other paths than expedited discharge, we estimated the association between hospital discharge tendency and mortality for hip fracture patients at the day of admission. Because few patients are discharged on the first day, any association would indicate an effect through other mechanisms.

Pressure to discharge may result in successively frailer patients being eligible for discharge on later days after admission. To assess whether our main analysis could be prone to bias due to such selection we computed the association between a hospital's discharge tendency averaged from day 2 to day 5 after admission and risk of death for all patients, regardless of day of discharge.

We also performed six variants of the instrumental variable analysis to test whether the results were sensitive to time adjustments, follow-up period, and age cut-off:
- Fridays were excluded to test whether associations were driven by the combination of increased probability for discharge on Fridays and less available resources in primary healthcare during weekends.

- We ran separate analyses before and after 2012, since 2012 was the starting point of a national reform with financial incentives intended to reduce length of stay in hospitals. $^{21}$

- By using 90 and 180 days of follow-up we assessed whether the results were sensitive to length of the risk period.

- By postponing follow-up by 1 or 5 days we tested whether the results were driven by death shortly after discharge.

- To test if the results were sensitive to age cut-offs, we performed the analysis for patients 50 years and older and 80 years and older.

- To test whether the results were sensitive to being eligible on a holiday/weekday we adjusted for this.

- To test if the results were sensitive for individual patient characteristics, we performed the analysis without such adjustment (age, sex, fracture of the femoral neck (S72.0), and previous hospitalizations within the last 2 calendar months).

\section{Results}

The study population is described in Table 1. During the study period the median length of stay for hip fracture patients was reduced from 7.5 days in 2008 to 5.0 days in 2016 .

Table I Number of Hospitalized Patients Eligible for Discharge per Day of Hospitalization, with Number of Discharges and Deaths. Descriptive Statistics (Age, Sex, Type of Fracture, and Pre-Admitted) on the Hospitalized Population

\begin{tabular}{|l|l|l|l|l|l|l|l|l|l|}
\hline $\begin{array}{l}\text { Day of } \\
\text { Hospitalization }\end{array}$ & $\begin{array}{l}\text { Number of } \\
\text { Hospitalized }\end{array}$ & $\begin{array}{l}\text { Died in } \\
\text { Hospital }\end{array}$ & $\begin{array}{l}\text { Eligible } \\
\text { for } \\
\text { Discharge }\end{array}$ & $\begin{array}{l}\text { Discharged } \\
\text { as Alive }\end{array}$ & $\begin{array}{l}\text { Discharged, } \\
\text { Dead } \\
\text { Within } \\
\text { 60 Days }\end{array}$ & $\begin{array}{l}\text { Average } \\
\text { Age } \\
\text { (Years) }\end{array}$ & $\begin{array}{l}\text { Female (\%) } \\
\text { Type of } \\
\text { Fracture } \\
\text { S72.0 } \\
\text { (\%) }\end{array}$ & $\begin{array}{l}\text { Pre- } \\
\text { Admitted, 60 } \\
\text { Days (\%) }\end{array}$ \\
\hline 1 & 64,914 & 52 & 64,862 & 16 & 4 & 84.7 & 72 & 60 & 19 \\
2 & 64,846 & 227 & 64,619 & 1406 & 324 & 84.7 & 72 & 60 & 19 \\
3 & 63,213 & 324 & 62,889 & 4950 & 934 & 84.6 & 72 & 59 & 19 \\
4 & 57,939 & 277 & 57,662 & 7367 & 1082 & 84.5 & 72 & 59 & 19 \\
5 & 50,295 & 215 & 50,080 & 8106 & 882 & 84.3 & 72 & 59 & 19 \\
6 & 41,974 & 198 & 41,776 & 7584 & 625 & 84.2 & 71 & 59 & 19 \\
7 & 34,192 & 181 & 34,011 & 6442 & 453 & 84.2 & 71 & 59 & 20 \\
8 & 27,569 & 134 & 27,435 & 5136 & 380 & 84.2 & 70 & 59 & 20 \\
9 & 22,299 & 142 & 22,157 & 3972 & 288 & 84.3 & 69 & 59 & 21 \\
10 & 18,185 & 100 & 18,085 & 3093 & 204 & 84.4 & 69 & 58 & 21 \\
11 & 14,992 & 77 & 14,915 & 2628 & 205 & 84.4 & 69 & 58 & 22 \\
12 & 12,287 & 67 & 12,220 & 1990 & 174 & 84.5 & 68 & 57 & 23 \\
13 & 10,230 & 57 & 10,173 & 1657 & 120 & 84.5 & 68 & 57 & 23 \\
14 & 8516 & 59 & 8457 & 1385 & 136 & 84.5 & 68 & 57 \\
\hline
\end{tabular}




\section{Initial Analysis}

In the initial analysis, as shown in Supplementary Figure S2, patients who were discharged within 4 days of admission had markedly higher 60 -day mortality (hazard ratio $=1.83$ on day 2, $95 \%$ confidence interval $(\mathrm{CI})=1.62-2.06)$ compared to patients who were not discharged on the same day. For patients who were discharged on day 6 or later, the corresponding mortality was lower among discharged patients compared to those who remained in hospital (hazard ratio $=0.70$ on day $6,95 \% \mathrm{CI}=0.64-0.77$ ).

\section{Instrumental Variable Analyses}

In the data material, an average of $24 \%$ (standard deviation $(\mathrm{SD})=8)$ of all acutely admitted patients were discharged per day, see Supplementary Figure S3. On Fridays the number was $30 \%(\mathrm{SD}=7)$. In the analysis of hospital discharge tendency and patient mortality, we found that mortality was 1.02-times higher $(95 \% \mathrm{CI}=1.01-1.03)$ per 10 percentage points increase in hospital discharge tendency, as shown in Figure 1. Except for days 7-9, all triplets of days showed higher mortality with higher hospital discharge tendency. The association between hospital discharge tendency and the probability of discharge of hip fracture patients is shown in Figure 2. The overall probability of discharge was 5.5 percentage points higher $(95 \% \mathrm{CI}=5.3-5.7)$ per 10 percentage points increase in hospital discharge tendency, with a partial F-statistic of 5,814. The probability of discharge was positively associated with discharge tendency for all triplets of days.

In the full instrumental variable analysis, we used discharges predicted by hospital discharge tendency to estimate the effect on risk of death. The results in Figure 3 show the estimated difference in risk of death between patients discharged from organizational causes and patients remaining in hospital. Overall, such discharges were associated with 3.7 percentage points higher risk of death within 60 days

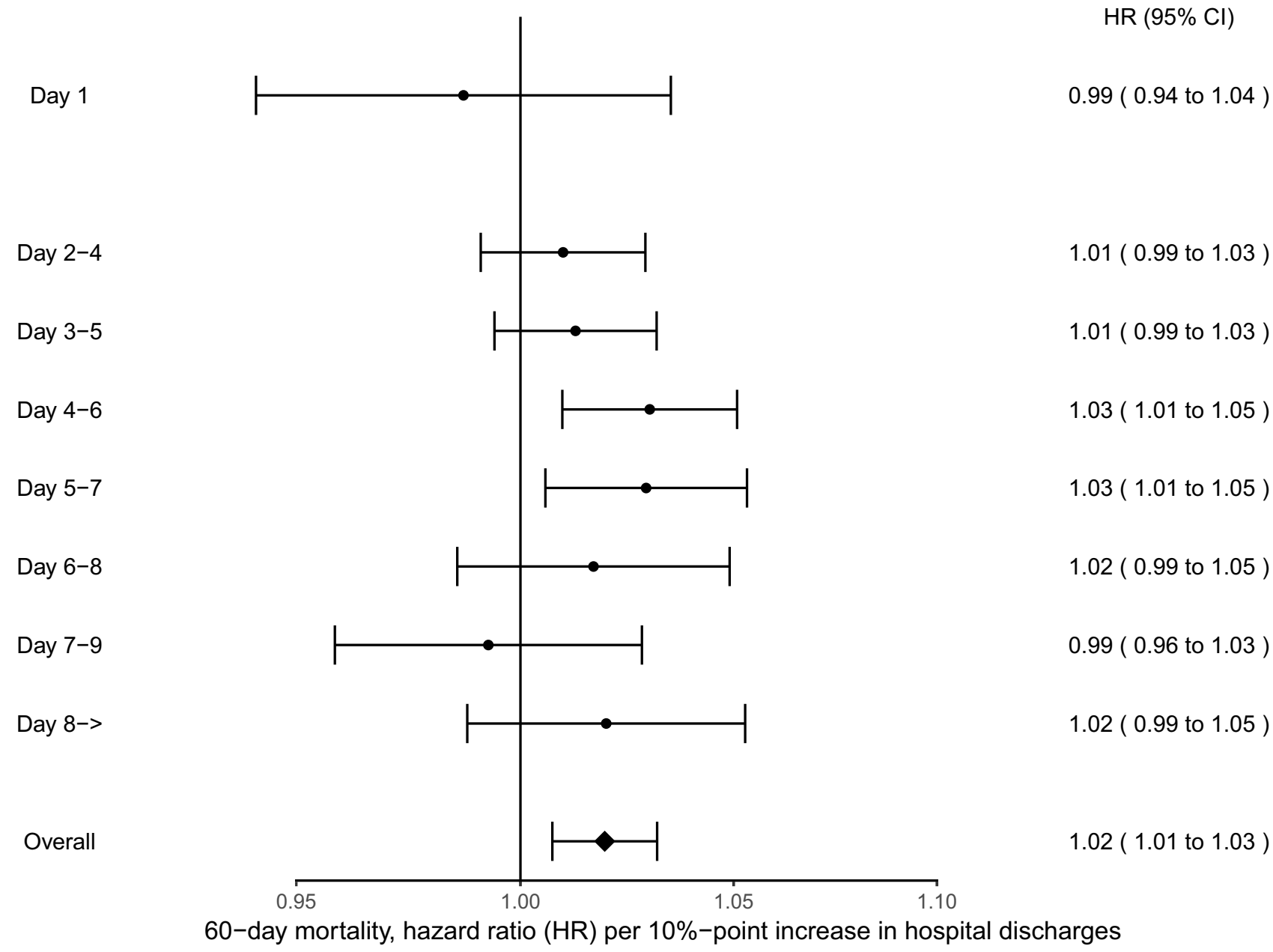

Figure I Hazard ratio (HR) for 60-day mortality per 10 percentage points increase in hospital discharges. Adjusted for day of hospitalization, holiday/weekend admissions, age, age squared, sex, S72.0 as primary diagnosis, prior admissions and discharges on previous days, and analysed within the same month, year, and hospital. 


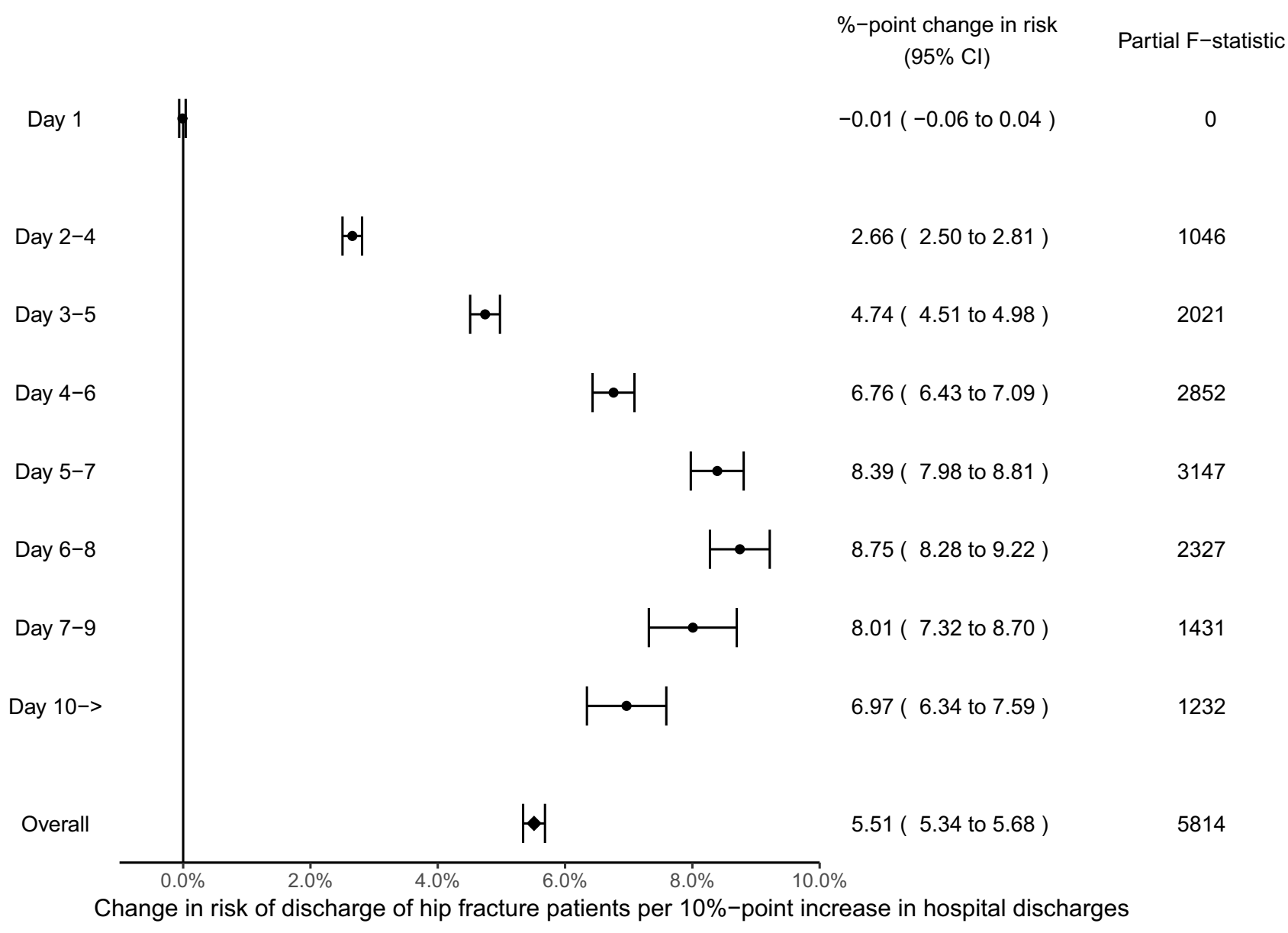

Figure 2 Change in absolute risk for discharge per 10 percentage points increase in hospital discharge tendency. Adjusted for day of hospitalization, holiday/weekend admissions, age, age squared, sex, S72.0 as primary diagnosis, prior admissions and discharges on previous days, and analysed within the same month, year, and hospital.

$(95 \% \mathrm{CI}=1.4-6.0)$. The same was true for all triplets of days after admission; the highest was observed at day 4-6 after admission, yielding a 4.2 percentage point higher risk of death $(95 \% \mathrm{CI}=1.3-7.2)$.

\section{Testing Instrumental Variable Assumption and Sensitivity Analyses}

The results of the tests of independence are illustrated in Figure 4 (numbers are presented in Supplementary Table S1). We observed no substantial associations between measured patient characteristics and hospital discharge tendency.

The sensitivity analysis of hospital discharge tendency and mortality at the day of admission showed no association $(\mathrm{HR}=0.99,95 \% \mathrm{CI}=0.94-1.04)$. The analysis of the association between a hospital's discharge tendency averaged from day 2 to day 5 after admission and risk of death for all patients yielded a hazard ratio of 1.08 (95\% $\mathrm{CI}=1.02-1.15)$. Figure 5 shows the overall estimated effect for the additional analyses, with no substantially different estimates from the main analysis.

\section{Discussion}

In this large study of hip fracture patients, we used observational data to mimic a series of clinical trials. Our aim was to assess whether discharges caused by factors that are independent of each patient's clinical condition may increase patient mortality. We estimated a 3.7 percentage points higher risk of death within 60 days for patients discharged from organizational causes.

In this study each day was analysed as a new trial for discharge, thereby avoiding bias due to in-hospital deaths. Initially, we compared mortality between discharged patients and patients who were not discharged on each given day after admission. The results showed an apparent drop in mortality for later discharges, suggesting a successive protective effect of discharges after day 5. This result is in line with the findings reported by a recent Swedish study, ${ }^{5}$ because late discharges in 


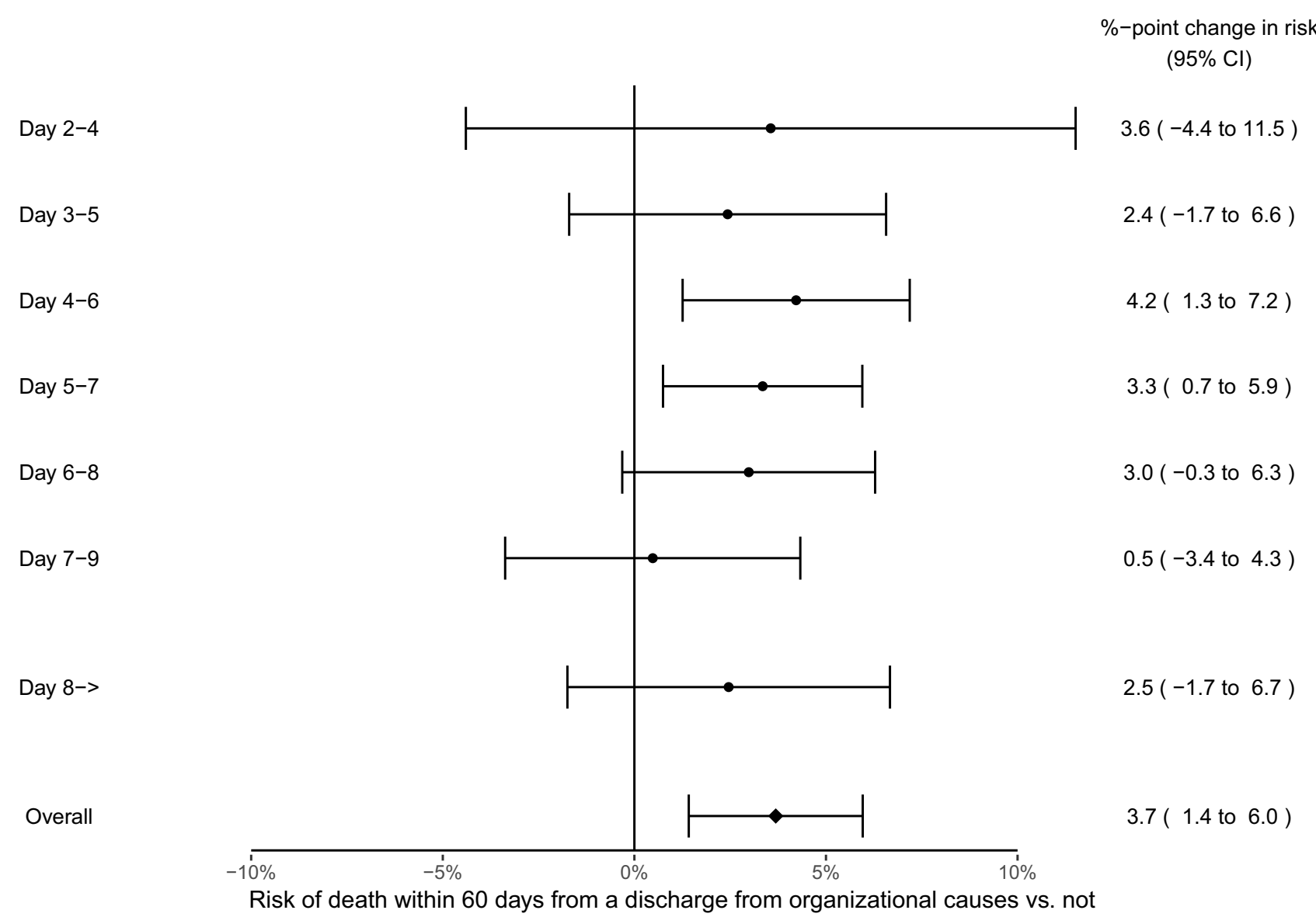

Figure 3 The effect of discharges from organizational causes on risk of death within 60 days, estimated with an instrumental variable analysis using two-stage ordinary least squares. Adjusted for day of hospitalization, holiday/weekend admissions, age, age squared, sex, S72.0 as primary diagnosis, prior admissions and discharge tendency on previous days. Analysed within the same month, year, and hospital.

our initial analysis roughly correspond to long hospitalizations in the Nordström analysis. However, both our initial analysis and the Nordström analysis are susceptible to bias due to confounding by indication for discharge. If our initial analysis actually suffers from bias depends on whether all indications for discharge are properly measured and adjusted for, but such a situation would not be realistic to expect.

In an attempt to overcome the potential for confounding by indication, we used an instrumental variable analysis, in which we relied on assumptions that differ from those of previous studies. Thus, we used the overall tendency to discharge patients with other acute conditions as an instrumental variable and assumed that this discharge tendency would randomly affect each hip fracture patient's probability of being discharged on a given day. Instead of having to rely on adjusting for differences in patient characteristics, we assessed the effect of an expedited discharge of hip fracture patients by using the variability from the hospital wide discharge tendency.

\section{Strength and Limitations}

By comparing effects of discharges among patients who were eligible for discharge at each day of hospitalization we avoided selection bias due to in-hospital deaths. Then, by using an instrumental variable approach we may avoid confounding by the indications for discharge without relying solely on adjustments for differences in patient characteristics.

Instrumental variable estimates require large data sets. This study achieved relatively precise estimates by considering all patients with hip fractures who were admitted to hospital in Norway between 2008 and 2016. Information about all other acute hospitalizations were used to compute the daily tendency to discharge for each Norwegian hospital during the study period. Deaths from any cause during 60 days of follow-up, not limited to in-hospital deaths, was available for every patient in data from the Norwegian Cause of Death Registry. ${ }^{22}$

Our findings were robust for different time adjustments, follow-up period, and using different cut-offs for 


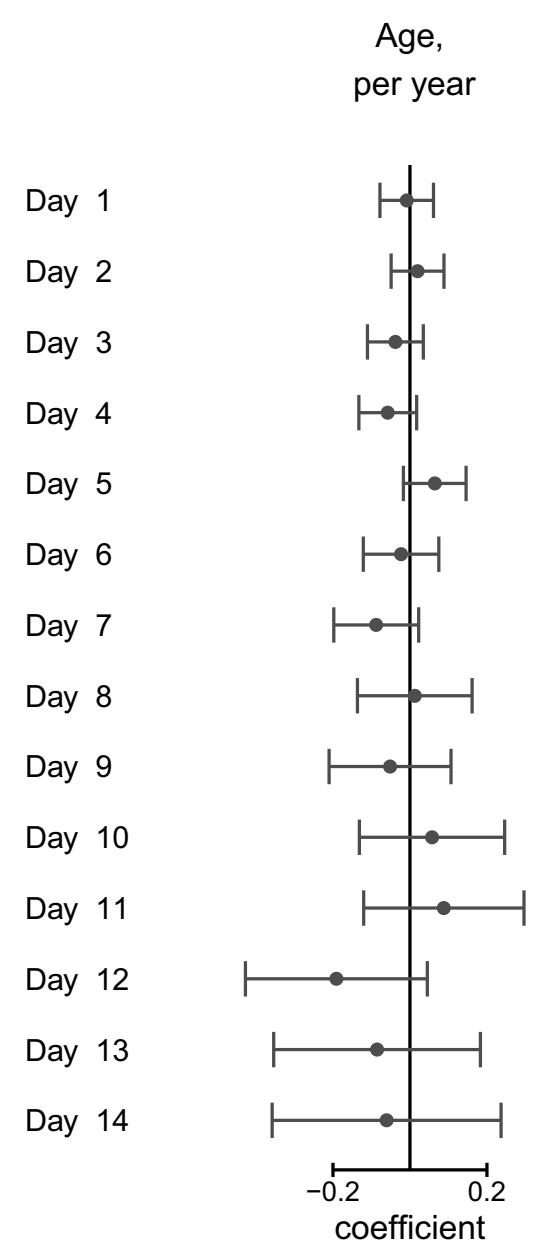

Woman
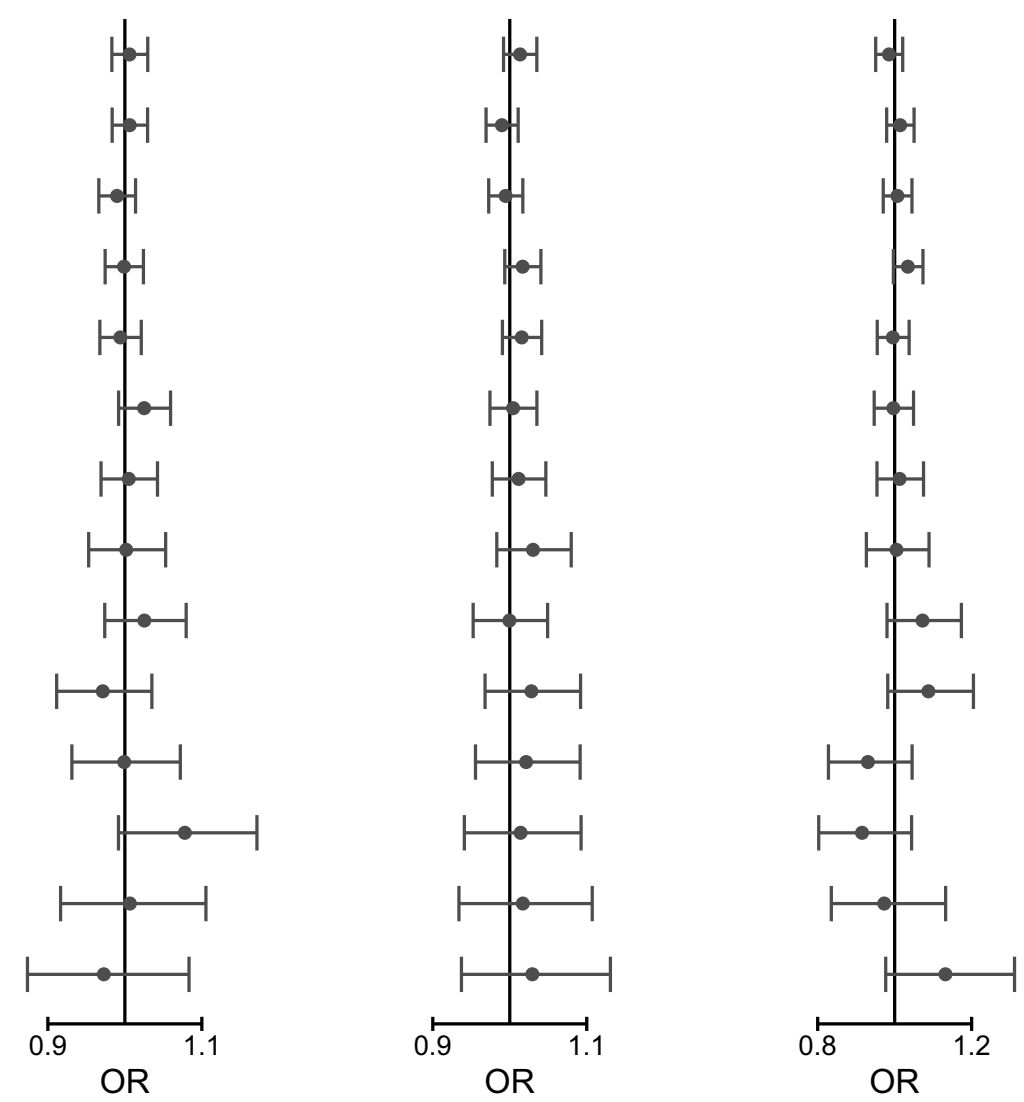

Figure 4 Tests of independence assumptions. Each column displays the estimates and $95 \% \mathrm{Cl}$ for the association between patient characteristics and hospital discharge tendency for all eligible patients at each day of hospitalization. Adjusted for holiday/weekend admissions, prior admissions, discharge tendency on previous days. Also adjusted for age, age squared, sex, S72.0 as primary diagnosis, if applicable. Analyzed within the same month, year, and hospital.

age. Our main analysis could be influenced by selection bias, since pressure to discharge may result in successively frailer patients being eligible for discharge on later days after admission. However, we found that analysing discharge tendency averaged from day 2 to day 5 and risk of death for all patients present at day 1 yielded similar results as the main analysis.

\section{Interpretation of the Results}

To be a valid instrument, hospital discharge tendency should satisfy a set of assumptions. ${ }^{16}$ First, it must have a sufficiently strong association with the individual hip fracture patient's probability of discharge. We found an F-value close to 6,000 , implying that this instrument relevance criterion was satisfied.

The tendency to discharge should also be independent of the patient's clinical indications for discharge. It is credible that this assumption is met, because a patient's clinical condition is unlikely to influence the tendency to discharge other patient groups in the hospital. We checked for violations of this independence assumption and found no apparent associations between measured patient characteristics and the hospital's tendency to discharge other patients.

To satisfy the exclusion restriction criterion, there should be no effect of the hospital's tendency to discharge on risk of death through other mechanisms than expedited discharge. This assumption is not possible to test directly, although day 1 of hospitalization can be considered for an indication of a violation. Since almost no patients were discharged on day 1, an association between discharge tendency and mortality would indicate an effect through alternative mechanisms. We found no such apparent association. Still, our findings could also be a result of other mechanisms, for example large volumes of discharges, which could create pressure in primary healthcare, or less 


\section{Full analysis}

Before 2012

After 2012

90 days follow-up

180 days follow-up

1 day postponed follow-up

5 days postponed follow-up

Excluding Fridays

Adjusted for day of hospitalization being holiday/weekday

Over 50 years old

Over 80 years old

No adjustments for patient characteristics

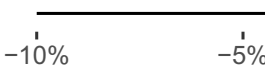

$-5 \%$

Risk of death within 60 days from a discharge from organizational causes vs. not
$3.7(1.4$ to 6.0$)$

$5.0(1.0$ to 9.0$)$

$2.4(-0.6$ to 5.3$)$

$3.5(1.0$ to 6.0$)$

3.5 ( 0.6 to 6.4$)$

$3.5(1.3$ to 5.8$)$

$2.1(0.0$ to 4.3$)$

$4.7(1.4$ to 8.0$)$

$7.5(1.4$ to 13.6$)$

$3.6(1.6$ to 5.6$)$

$3.8(1.1$ to 6.5$)$

$3.4(1.3$ to 5.4$)$

Figure 5 Sensitivity analyses. Overall effect estimates for the full instrumental variable analysis with different cut-offs in time, follow-up, and age groups. Adjusted for day of hospitalization, holiday/weekend admissions, age, age squared, sex, S72.0 as primary diagnosis, prior admissions and discharges on previous days, and analysed within the same month, year, and hospital.

rigorous discharge routines. In cases of rapid deterioration, e.g., due to infections or other complications after orthopedic surgery, hospitalized patients will have sooner access to essential emergency care than discharged patients. It is also possible that hospitals could provide better recovery after surgery than primary healthcare, securing a better long-term prognosis.

The estimate of increased risk of death following an expedited discharge should be considered an effect for an unobserved group of patients sensitive for discharge pressure (a local average treatment effect, LATE). ${ }^{23}$ The estimated effect thus concerns patients whose discharge decision is actually influenced by the pressure to discharge, presumably patients that would have stayed if the discharge pressure was lower.

One purpose of our analysis was to shift focus from length of stay to the process of discharge, because discharge may be a more relevant target for preventive actions. Also, discharge could be a realistic aim for a potential intervention study, for example, using alternative discharge routines as an intervention. The discharge process is key to patient safety because this is when important information about medication, rehabilitation, and follow-up should be given to the patient, sent to the primary healthcare services, the family, and other relevant caregivers. In light of our findings, an adequate clinical evaluation of the timing of discharge may be a useful approach to gain further insight into the safety of frail patient groups. Such studies have been done in the past, for example regarding orthopedic care combined with comprehensive geriatric care, which has been shown to improve the timing of discharge of hip fracture patients. ${ }^{24-26}$

\section{Conclusion}

In this study we found that hospital discharges were associated with both expedited discharges and risk of death for Norwegian hip fracture patients. This may indicate that expedited discharges are harmful. 


\section{Funding}

SMN an KSA were funded by the Norwegian Research Council with grant number 250335, JHB and AA were funded with grant number 295989.

\section{Disclosure}

The authors report no conflicts of interest in this work.

\section{References}

1. Eurostat. Hospital discharges and length of stay statistics 2018. Available from: https://ec.europa.eu/eurostat/statistics-explained/index.php/ Hospital_discharges_and_length_of_stay_statistics. Accessed February 7, 2020.

2. Cram P, Rush RP. Length of hospital stay after hip fracture. $B M J$. 2015;350. doi:10.1136/bmj.h823

3. Bueno H, Ross JS, Wang Y, et al. Trends in length of stay and short-term outcomes among Medicare patients hospitalized for heart failure, 1993-2006. JAMA. 2010;303(21):2141-2147. doi:10. 1001/jama.2010.748

4. Lewis PM, Waddell JP. When is the ideal time to operate on a patient with a fracture of the hip? A review of the available literature. Bone Joint J. 2016;98-B(12):1573-1581. doi:10.1302/0301-620X.98B12. BJJ-2016-0362.R2

5. Nordstrom P, Gustafson Y, Michaelsson K, et al. Length of hospital stay after hip fracture and short term risk of death after discharge: a total cohort study in Sweden. BMJ. 2015;350:h696. doi:10.1136/ bmj.h696

6. Nikkel LE, Kates SL, Schreck M, et al. Length of hospital stay after hip fracture and risk of early mortality after discharge in New York state: retrospective cohort study. BMJ. 2015;351:h6246. doi:10.1136/ bmj.h6246

7. Johnsen SP, Kristensen PK. Link between length of hospital stay and mortality among hip fracture patients varies across healthcare systems. Evid Based Nurs. 2017;20(1):21. doi:10.1136/eb-2016102348

8. Pouw ME, Peelen LM, Moons KGM, et al. Including post-discharge mortality in calculation of hospital standardised mortality ratios: retrospective analysis of hospital episode statistics. BMJ. 2013;347: f5913. doi:10.1136/bmj.f5913

9. Suissa S. Immortal time bias in pharmacoepidemiology. Am J Epidemiol. 2007;167(4):492-499. doi:10.1093/aje/kwm324

10. Hesselink G, Flink M, Olsson M, et al. Are patients discharged with care? A qualitative study of perceptions and experiences of patients, family members and care providers. BMJ Qual Saf. 2012;21(Supp1 1):i39-i49. doi:10.1136/bmjqs-2012-001165

11. Hernán MA, Alonso A, Logan R, et al. Observational studies analyzed like randomized experiments: an application to postmenopausal hormone therapy and coronary heart disease. Epidemiology. 2008;19 (6):766-779. doi:10.1097/EDE.0b013e3181875e61

Clinical Epidemiology

\section{Publish your work in this journal}

Clinical Epidemiology is an international, peer-reviewed, open access, online journal focusing on disease and drug epidemiology, identification of risk factors and screening procedures to develop optimal preventative initiatives and programs. Specific topics include: diagnosis, prognosis, treatment, screening, prevention, risk factor modification,

Submit your manuscript here: https://www.dovepress.com/clinical-epidemiology-journal
12. Greenland S. An introduction to instrumental variables for epidemiologists. Int J Epidemiol. 2000;29(4):722-729. doi:10.1093/ ije/29.4.722

13. Bay-Nielsen H NOMESCO Classification of Surgical Procedures: nordic Medico-Statistical Committee (NOMESCO); 2010. Available from: https://norden.diva-portal.org/smash/get/diva2:970547/ FULLTEXT01.pdf. Accessed February 7, 2020.

14. Høiberg MP, Gram J, Hermann P, et al. The incidence of hip fractures in Norway - accuracy of the national Norwegian patient registry. BMC Musculoskelet Disord. 2014;15:372. doi:10.1186/1471-247415-372

15. Hernán MA, Robins JM. Using big data to emulate a target trial when a randomized trial is not available. Am $J$ Epidemiol. 2016;183 (8):758-764. doi:10.1093/aje/kwv254

16. Davies NM, Smith GD, Windmeijer F, et al. Issues in the reporting and conduct of instrumental variable studies: a systematic review. Epidemiology. 2013;24(3):363-369. doi:10.1097/EDE.0b013e3182 8 abafb

17. Davies NM, Holmes MV, Davey Smith G. Reading Mendelian randomisation studies: a guide, glossary, and checklist for clinicians. BMJ. 2018;362:k601. doi:10.1136/bmj.k601

18. Rassen JA, Schneeweiss S, Glynn RJ, et al. Instrumental variable analysis for estimation of treatment effects with dichotomous outcomes. Am J Epidemiol. 2008;169(3):273-284. doi:10.1093/aje/ kwn299

19. Danaei G, Rodríguez LAG, Cantero OF, et al. Observational data for comparative effectiveness research: an emulation of randomised trials of statins and primary prevention of coronary heart disease. Stat Methods Med Res. 2013;22(1):70-96. doi:10.1177/0962280211403603

20. Fleischman RJ, Adams AL, Hedges JR, et al. The optimum follow-up period for assessing mortality outcomes in injured older adults. $J \mathrm{Am}$ Geriatr Soc. 2010;58(10):1843-1849. doi:10.1111/j.1532-5415.2010. 03065.x

21. Norwegian Ministry of Health \& Care services. Report No. 47 to the Storting (2008-2009). The Coordination Reform - Proper treatment at the right place and right time. Available from: http://www.regjerin gen.no/nb/dep/hod/dok/regpubl/stmeld/2008-2009/stmeld-nr-472008-2009-.html?id=567201. Accessed February 7, 2020.

22. Norwegian Cause of Death Registry: Norwegian Institute of Public Health. Available from: https://www.fhi.no/en/hn/health-registries /cause-of-death-registry/. Accessed February 7, 2020.

23. Hernán MARJ. Causal Inference. Boca Raton: Chapman \& Hall/ CRC;2019.

24. Prestmo A, Hagen G, Sletvold O, et al. Comprehensive geriatric care for patients with hip fractures: a prospective, randomised, controlled trial. Lancet. 2015;385(9978):1623-1633. doi:10.1016/S0140-6736(14) 62409-0

25. Chudyk AM, Jutai JW, Petrella RJ, et al. Systematic review of hip fracture rehabilitation practices in the elderly. Arch Phys Med Rehabil. 2009;90(2):246-262. doi:10.1016/j.apmr.2008.06.036

26. Ellis G, Whitehead MA, Robinson D, et al. Comprehensive geriatric assessment for older adults admitted to hospital: meta-analysis of randomised controlled trials. BMJ. 2011;343:d6553. doi:10.1136/ bmj.d6553

systematic reviews, risk \& safety of medical interventions, epidemiology \& biostatistical methods, and evaluation of guidelines, translational medicine, health policies \& economic evaluations. The manuscript management system is completely online and includes a very quick and fair peer-review system, which is all easy to use. 